\title{
Expression of Polypeptide $N$-Acetylgalactosaminyltransferase-6 in Epithelial Ovarian Carcinoma
}

\author{
MIDORI MURAKAMI, SEIJI KAGAMI, THUY THI NGUYEN, CHIHO KOI, \\ TOMOKO KURITA and TORU HACHISUGA \\ Department of Obstetrics and Gynecology, \\ University of Occupational and Environmental Health, Kitakyushu, Japan
}

\begin{abstract}
Background: The family of polypeptide $\mathrm{N}$ acetylgalactosanimyltransferases (GalNAc-Ts) are important factors in glycosylation in carcinomas. The purpose of this study was to investigate the clinical significance of GalNAcT6 and its correlation with the prognosis of epithelial ovarian carcinoma. Materials and Methods: A total of 150 patients with epithelial ovarian carcinoma were enrolled and the relationship between GalNAc-T6 expression by immunohistochemistry and long-term survival was evaluated. Results: The expression of GalNAc-T6 was positive in $57.6 \%$ (34/59) of those with serous carcinoma, 85.3\% (29/34) in mucinous carcinoma, $15.6 \%$ (5/27) in clear cell carcinoma, and $44 \%$ (14/25) in endometrioid carcinoma. In a KaplanMeier analysis of patients with grade 1 or 2 serous carcinoma, the 10-year overall survival rates were $47.4 \%$ in the GalNAc-T6-positive and $9.1 \%$ in the GalNAc-T6negative groups $(p=0.047)$. Conclusion: GalNAc-T6 expression in epithelial ovarian carcinoma was different according to pathological type. In low-grade serous carcinoma, GalNAc-T6 expression may contribute to improved long-term survival.
\end{abstract}

Epithelial ovarian carcinoma is the leading cause of death from gynecological cancer and the morbidity has been increasing in recent years. Increasing obesity is one of the reasons for this (1). Epithelial ovarian carcinoma is responsible for 8,300 new cases per year and 4,600 deaths per year in Japan (2). Because of few subjective symptoms in the early stage, ovarian carcinoma is often diagnosed at an advanced stage. Surgery and chemotherapy have become

Correspondence to: Dr. Seiji Kagami, Depertment of Obstetrics and Gynecology, University of Occupational and Environmental Health, Japan, 1-1, Iseigaoka, Yahatanishi-ku, Kitakyushu, Fukuoka, 8078555, Japan. Tel: +81 936031611, e-mail: s-kagami@med.uoeh-u.ac.jp

Key Words: Ovarian carcinoma, serous ovarian carcinoma, GalNAc-T6. better, but long-term survival rates for patients with advanced disease are still low $(3,4)$.

The family of polypeptide $N$-acetylgalactosanimyltransferases (GalNAc-Ts) are responsible for the altered glycosylation in carcinoma. In a review, several studies were reported to have found GalNAc-Ts to be associated with the functional alteration of cancer cells, including their antigenic and adhesive properties, as well as their potential for invasion and metastasis (5). It has been reported that there is a relationship between GalNAc-T6 and clinicopathological factors in breast (6) and pancreatic (7) cancer. GalNAc-T6 is expected to be a marker of glycosylation modifications in carcinoma. In this study, we investigated GalNAc-T6 in epithelial ovarian carcinoma.

\section{Materials and Methods}

Patients and tumor samples. The subjects of the present study were 150 patients with epithelial ovarian carcinoma who received treatment at the University of Occupational and Environmental Health Hospital (Kitakyusyu, Japan) between January 1995 and July 2008. Fifty-nine of these patients had serous carcinoma, 34 had mucinous carcinoma, 32 clear cell carcinoma, and 25 endometrioid carcinoma. All patients underwent cytoreductive surgery and chemotherapy with carboplatin and paclitaxel. We used paraffin-embedded tissue blocks from primary surgery for immunohistochemical study. Clinical information was gathered from the patient's records retrospectively. The study was approved by the Review Board of our hospital on Ethical Issues (H27071)

Immunohistochemical analysis. Expression of GalNAc-T6 were analyzed immunohistochemically in paraffin-embedded tumor samples. Histological sections of $4 \mu \mathrm{m}$ were deparaffinised and rehydrated. Antigen retrieval was carried out at $121^{\circ} \mathrm{C}$ for $3 \mathrm{~min}$. The sections were incubated with polyclonal antibody to GalNAcT6 (DAKO, Kyoto, Japan) (1:1600) for $1 \mathrm{~h}$ at room temperature. Antibody binding was visualized using Envision+ Dual link system (DAKO, Kyoto, Japan). Sections were counterstained with hematoxylin and mounted. Slides were evaluated by two independent authors who were blinded to the clinicopathological data and patient outcomes. Scoring was categorized as 0 (negative) to 3 (very strong), and subsequently grouped into negative (score 0 
Table I. Clinicopathological variables by subtype of epithelial ovarian carcinoma.

\begin{tabular}{|c|c|c|c|c|c|}
\hline Variable & Subgroup & $\begin{array}{c}\text { Serous, } \mathrm{n} \\
\mathrm{n}=59\end{array}$ & $\begin{array}{c}\text { Mucinous, } \mathrm{n} \\
\mathrm{n}=34\end{array}$ & $\begin{array}{c}\text { Clear cell, } n \\
n=32\end{array}$ & $\begin{array}{c}\text { Endometrioid, } \mathrm{n} \\
\mathrm{n}=25\end{array}$ \\
\hline \multirow[t]{2}{*}{ Age } & $\leq 60$ Years & 32 & 23 & 27 & 19 \\
\hline & $>60$ Years & 27 & 11 & 5 & 6 \\
\hline \multirow[t]{2}{*}{ Grade } & 1,2 & 30 & 33 & 0 & 21 \\
\hline & 3 & 29 & 1 & 32 & 4 \\
\hline \multirow[t]{2}{*}{ FIGO stage } & I, II & 7 & 30 & 23 & 22 \\
\hline & III, IV & 52 & 4 & 9 & 3 \\
\hline \multirow[t]{2}{*}{ LN metastasis } & Negative & 32 & 31 & 29 & 25 \\
\hline & Positive & 27 & 3 & 3 & 0 \\
\hline \multirow[t]{2}{*}{ Surgery } & Optimal & 25 & 30 & 26 & 21 \\
\hline & Suboptimal & 34 & 4 & 6 & 4 \\
\hline \multirow[t]{3}{*}{ GalNAc-T6 } & Negative & 25 & 5 & 27 & 14 \\
\hline & Positive & 34 & 29 & 5 & 11 \\
\hline & Positivity rate & $57.6 \%$ & $85.3 \%$ & $15.6 \%$ & $44.0 \%$ \\
\hline
\end{tabular}

LN: Lymph node; GalNAc-T6: $N$-acetylgalactosaminyltransferase-6.

and 1) and positive (score 2 and 3) groups is order to analyze the significance of GalNAc-T6 expression.

Statistical analysis. Differences in survival by variable were evaluated using the log-rank test, whereas Cox's proportional hazards model was used to identify, and simultaneously evaluate, any independent prognostic factors associated with relative survival. A value of $p<0.05$ was considered significant. The cumulative 10 year survival was determined using the Kaplan-Meier method. The statistical analyses were performed using SPSS for Windows version 20.0 (IBM Corp., Armonk, NY, USA).

\section{Results}

Patient characteristics. As shown in Table I, the study included 150 patients with clinicopathological features representative of ovarian epithelial carcinoma. The average age at surgery was 59.5 years in those with serous carcinoma, 49.9 years in those with mucinous carcinoma, 49.5 years in those with clear cell carcinoma, and 56.5 years in those with endometrioid carcinoma. Based on International Federation of Gynecology and Obstetrics (FIGO) stage, $88 \%$ of patients had advanced stage in those with serous carcinoma, but the majority of patients had early stage disease for the other three pathological subtypes.

GalNAc-T6 expression in ovarian epithelial carcinoma. Immunohistochemically, GalNAc-T6 expression was positive in $57.6 \%$ (34/59) of those with serous carcinoma, $85.3 \%$ (29/34) in mucinous carcinoma, $15.6 \%(5 / 27)$ in clear cell carcinoma, and $44.0 \%(11 / 25)$ in endometrioid carcinoma (Table I). Characteristically, mucinous carcinoma was strongly positive, whilst clear cell carcinoma was negative or very weakly stained (Figure 1). GalNAc-T6 expression was positive in normal fallopian epithelium, and benign serous tumor (Figure 2).
Association of GalNAc-T6 expression with clinicopathological variables. There was no significant difference of GalNAc-T6 expression regarding age, grade, FIGO stage, lymph node metastasis, and optimal surgery in those with serous ovarian carcinoma.

Correlation between GalNAc-T6 expression and prognosis. The 10-year overall survival rate for patients with serous ovarian carcinoma was $26.9 \%$ for the GalNAc-T6-positive group and $24.5 \%$ for the GalNAc-T6-negative group $(p=0.493)$ (Figure 3A). In subgroup analysis, for those with grade 1 or 2 serous carcinoma, the 10 - year overall survival rate was $47.4 \%$ in the GalNAc-T6-positive and $9.1 \%$ in the GalNAc-T6-negative group $(p=0.047)$ (Figure 3B).

\section{Discussion}

Many studies have reported the roles of GalNAc-Ts in carcinoma. Previous research revealed that GalNAc-T6 expression was related to some kinds of carcinomas. In breast cancer, GalNAc-T6 expression was statistically significantly associated with $\mathrm{T} 1$ tumor stage (6). In pancreatic cancer, GalNAc-T6 expression was related to good pathological differentiation, small tumor size, absence of vascular invasion, and low clinical stage (7). On the other hand, GalNAc-T6 expression correlated with advanced TNM stage, and independently predicted worse overall survival for patients with lung adenocarcinoma (8). Expression of GalNAc-T6 and its effects differ according to tissue type and specificity has not been confirmed.

In epithelial ovarian carcinoma, the expression of GalNAc-T3 correlated with shorter progression-free survivaI (9). GalNAc-T6 is considered an important glycosylation 

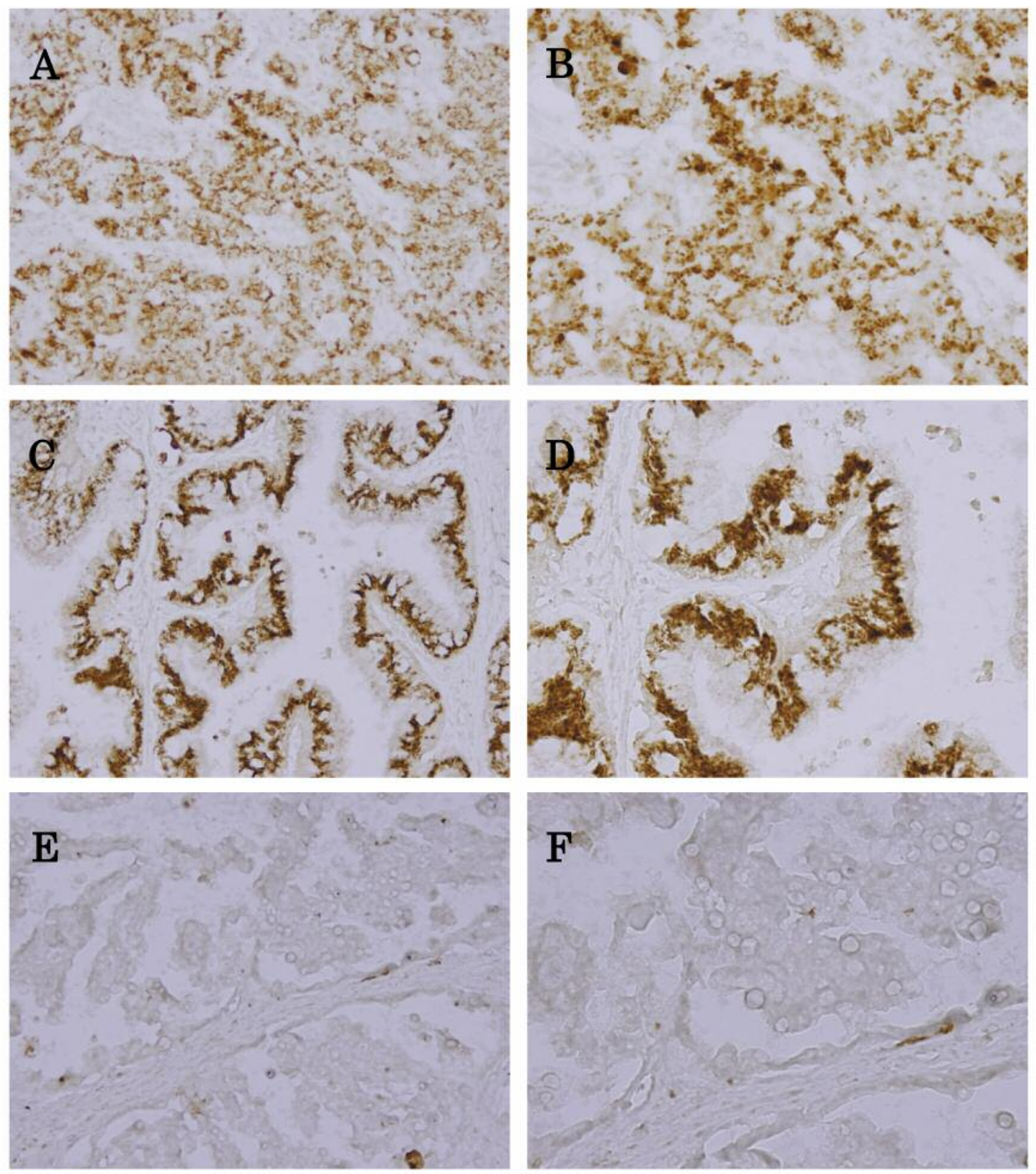

Figure 1. Immunohistochemical analysis of $\mathrm{N}$-acetylgalactosaminyltransferase-6 in epithelial ovarian carcinoma. Representative examples of serous carcinoma $(A: \times 100, B: \times 400)$, mucinous carcinoma $(C: \times 100, D: \times 400)$, and clear cell carcinoma $(E: \times 100, F: \times 400)$.

modification for cell-adhesion factors. The extent of dissemination and metastasis are essential factors for ovarian carcinoma prognosis. However, to our knowledge, there is no report of the relationship between GalNAc-T6 and ovarian epithelial carcinoma prognosis.

In this study, firstly, we confirmed the expression of GalNAc-T6 in four representative tissue types of ovarian carcinoma. Secondly, we performed a prognostic analysis of serous carcinoma. When examining the staining of GalNAc-T6 by pathological type, mucinous carcinoma, which is well-differentiated and has a good prognosis stained positively for GalNAc-T6 in $85.3 \%$ of cases, while in clear cell carcinoma, which is poorly differentiated and has a poor prognosis, $15.6 \%$ of cases were positive. From the above, this study suggests that the expression of GalNAc-T6 is different for each tissue type of ovarian 

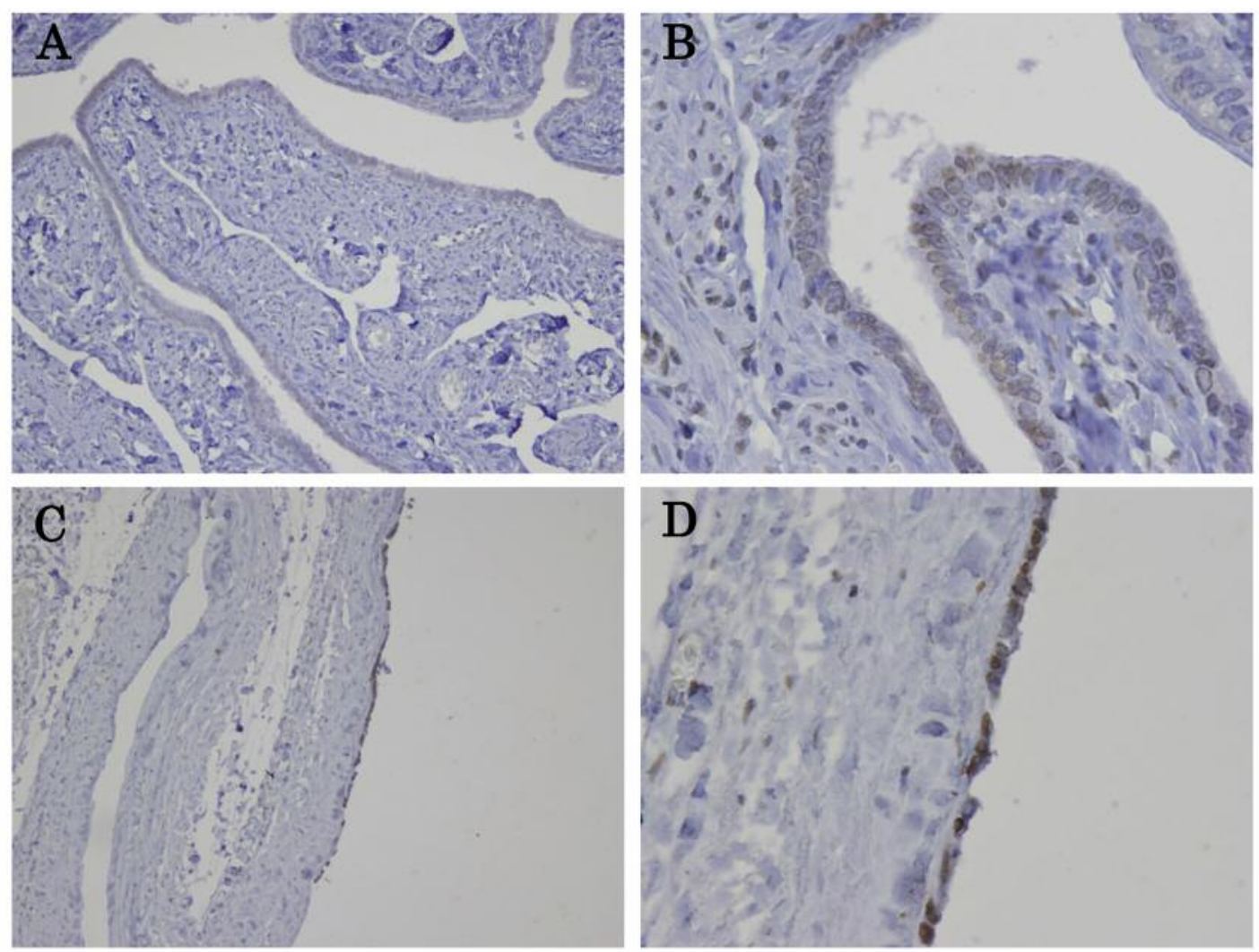

Figure 2. Immunohistochemical analysis of $N$-acetylgalactosaminyltransferase- 6 in fallopian tube and serous cyst. Representative examples of staining of fallopian tube $(A: \times 100, B: \times 400)$ and serous cyst $(C: \times 100, D: \times 400)$.

A

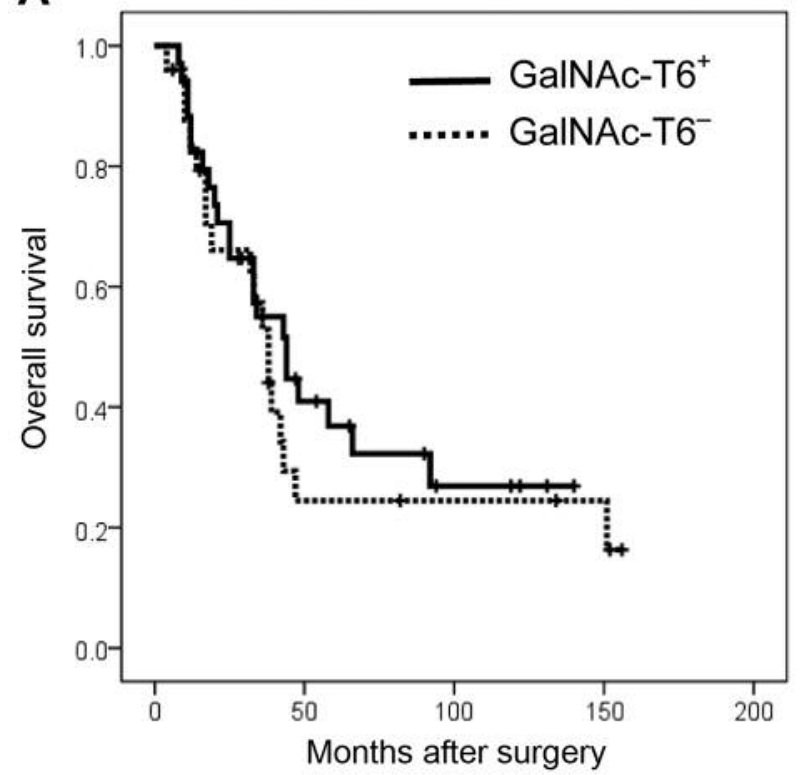

B

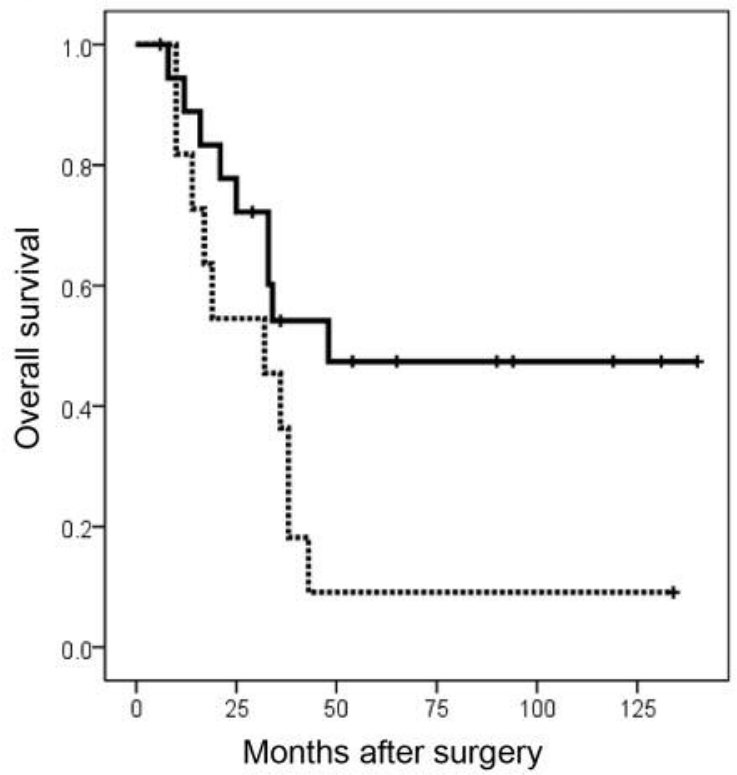

Figure 3. Kaplan-Meier curves of overall survival after surgery for all patients with serous ovarian carcinoma (A) and those with grade 1 or 2 serous carcinoma $(B)$. In the group with grade 1 or 2 serous carcinoma, patients with positive expression of GalNAc-T6 had significantly longer survival than those with negative expression ( $p=0.047)$. 
epithelial carcinoma. In serous carcinoma, there was no association between GalNAc-T6 expression and long-term prognosis. However, in grade 1 and 2 serous carcinoma, the group with positive expression of GalNAc-T6 had a significantly better prognosis.

Serous ovarian carcinoma can be classified into two categories depending on differentiation, and each pathogenesis is different. High-grade serous carcinoma has TP53 mutation and rapid progression. In contrast, low-grade serous carcinoma has been hypothesized to arise from a serous borderline tumor in a slow step-wise fashion $(3,10)$.

In this study, almost all benign serous tumors were GalNAc-T6-positive, while positivity for GalNAc-T6 in serous carcinoma was $57 \%$. The group negative for GalNAcT6 had a worse prognosis, therefore loss of GalNAc-T6 expression might contribute to acquire dysfunction in glycosylation.

In order to discuss the role of glycosylation, a larger number of cases should be accumulated and the relationship between expression of GalNAc-T6 and various factors of cell adhesion should be evaluated. Distinct progression of ovarian carcinoma is through dissemination, and the epithelial-mesenchymal transition plays a paramount role in dissemination and resistance to platinum chemotherapy. The relationship of GalNAc-T6 expression with these factors should be the focus of future molecular biological studies.

The results of this study suggested that expression of GalNAc-T6 in ovarian epithelial carcinoma differs by pathological subtype, and in relation to clinicopathological features. GalNAc-T6 expression might contribute to longterm survival in patients with grade 1 or 2 serous carcinoma. GalNAc-T6 may be a promising supplementary marker in ovarian carcinoma prognosis and help determine its management in the future.

\section{Acknowledgements}

The Authors thank the staff at Department of Pathology and Oncology of University of Occupational and Environmental Health, Japan for collecting past formalin-fixed paraffin-embedded specimens.

\section{References}

1 Anastasi E, Capoccia D, Granato T, Silecchia G, Rizzello M, Porpora MG, Frati L, Angeloni A and Leonetti F: Implementing the risk of ovarian malignancy algorithm adding obesity as a predictive factor. Anticancer Res 36: 6425-6429, 2016.

2 Haruta S, Furukawa N, Yoshizawa Y, Tsunami T, Nagai A, Kawaguchi R, Tanase Y, Yoshida S and Kobayashi H: Molecular genetics and epidemiology of epithelial ovarian cancer (Review). Oncol Rep 26: 1347-1356, 2011

3 Kurman RJ and Shih LM: Molecular pathogenesis and extraovarian origin of epithelial ovarian cancer. Shifting the paradigm. Hum Pathol 42(7): 918-931, 2011.

4 Bertelli G, Drews F and Lutchman-singh K: Bevacizumab for ovarian cancer at high risk of progression: reproducibility of trial results in 'Real-world' patients. Anticancer Res 36(9): 49474950, 2016.

5 Brockhausen I: Mucin-type O-glycans in human colon and breast cancer: glycodynamics and functions. EMBO Rep 7(6): 599-604, 2006.

6 Berois N, Mazal D, Ubillos L, Trajetenberg F, Nicolas A, Garau $\mathrm{XS}$, Magdelenat $\mathrm{H}$ and Osinaga E: UDP- $N$-Acetyl-Dgalactosamine polypeptide $N$-acetylgalactosaminyltransferase- 6 as a new immunohistochemical breast cancer marker. J Histochem Cytochem 54(3): 317-328, 2006.

$7 \mathrm{Li} Z$, Yamada S, Inenaga S, Imamura T, Wu Y, Wang KY, Shimagiri S, Nakano R, Izumi H, Kohno K and Sasaguri Y: Polypeptide $\mathrm{N}$-acetylgalactosaminyltransferase 6 expression in pancreatic cancer is an independent prognostic factor indicating better overall survival. Br J Cancer 104(12): 1882-1889, 2011.

8 Li Z, Yamada S, Wu Y, Wang KY, Liu YP, Uramoto H, Kohno K and Sasaguri Y: Polypeptide $N$-acetylgalactosaminyltransferase 6 expression independently predicts poor overall survival in patients with lung adenocarcinoma after curative resection. Oncotarget 7: 54463-54473, 2016.

9 Wang ZQ, Bachvarova M, Morin C, Plante M, Gregoire J, Renaud MC, Sebastianelli A and Bachvarov D: Role of the polypeptide $N$-acetylgalactosaminyltransferase 3 in ovarian cancer progression: possible implications in abnormal mucin $O$ glycosylation. Oncotarget 5: 544-560, 2014.

10 Vang R, Shih leM and Kurman RJ: Fallopian tube precursors of ovarian low- and high-grade serous neoplasms. Histopathology 62(1): 44-58, 2013.

Received May 2, 2017

Revised May 22, 2017

Accepted May 23, 2017 